\title{
Limits on Stability of Positive Molecular Ions in a Homogeneous Magnetic Field
}

\section{S. Vugalter ${ }^{\star}$}

Steklov Mathematical Institute, St. Petersburg and Radiophysical Research Institute, B. Pecherskaya 25/14, N. Novgorod, 603600, Russia

E-mail: simeon@nirfi.nnov.su

Received: 6 September 1995/Accepted: 12 February 1996

\begin{abstract}
The problem of stability of positive diatomic molecular ions with the nuclear charges $Z_{1}$ and $Z_{2}$ and $N$ electrons in a homogeneous magnetic field $B$ is studied for $Z_{1}, Z_{2}, N, B \rightarrow \infty$. The conditions of instability are obtained for different relations among $Z_{1}, Z_{2}, N$ and $B$. A new version of the HVZ theorem for systems in a homogeneous magnetic field is proved.
\end{abstract}

\section{Introduction}

It is well known [5] that a positive diatomic molecular ion with $N$ electrons and nuclear charges $Z_{1}$ and $Z_{2}$ is unstable for large $Z_{1}$ and $Z_{2}$. It means that for every positive $N$ there exists a constant $Z^{c}>0$ such that whenever both $Z_{1}>Z^{c}$ and $Z_{2}>Z^{c}$, the hamiltonian of the system (after the separation of the center of mass motion) has no discrete spectrum. Furthermore

$$
\varlimsup_{N \rightarrow \infty} Z^{c} N^{-1} \leqq 2 \text {. }
$$

In this paper we discuss a generalization of this result to molecules in a magnetic field. This case is more complicated for the following reasons. First we cannot use the standard definition of stability, because the center of mass motion cannot be separated in a homogeneous magnetic field in a standard sense. A key to this problem is a new version of the $H V Z$ theorem for the hamiltonian on the subspace of functions with fixed rotational $S O(2)$ symmetry which is proved in Sect. A1 (see Appendix). The idea of fixing of the type of the $S O(2)$ symmetry as a substitute for the separation of the center of mass motion for the $H V Z$ theorem was suggested earlier by G. Zhislin and the author [6]. But in [6] the $H V Z$ theorem was proved only for the case when either all the charges of the particles have the same sign or the magnetic field $B$ increases at infinity.

In this paper we consider molecular ions consisting of positive nuclei and negative electrons in a homogeneous magnetic field and the results of [6] can not be applied.

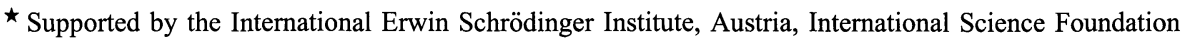
Grant N R 94000 and Grant of Russian Fond Fundament Issled. 94-01-01376
} 
Furthermore the physical intuition (and the idea of the proof) for the $H V Z$ theorem to hold in this case are different than in [6].

Another problem is the estimation of the energy of the system in a region where the distance between nuclei is small. For this goal, the "united atom estimate" was used in [5], but in a magnetic field this estimate is not optimal. To solve this problem some additional decompositions of the electron's configuration space and some new estimates are suggested in this paper.

Notice that a magnetic field can play an important role in stability, because it tries to localize particles in two dimensions. We take it into account in our study by choosing all the sizes of subregions of the configuration space to depend not only on the nuclei charges as in [5], but also on the magnetic field.

The main result of this paper is the statement that if the magnetic field $B$ is "weak" in the sense that for some $\delta_{0}>0$,

$$
\left(B Z_{2}^{-2}\right)^{3 / 2}\left[\ln \left(1+B Z_{2}^{-2}\right)\right]^{3+\delta_{0}}<Z_{1}
$$

and $Z_{2} \geqq Z_{1}$, then inequality (1.1) for critical charge $Z^{c}$ holds in the magnetic field case. We estimate also limits of stability in cases where (1.2) does not hold.

\section{Definitions and Results}

Let $D_{1}$ be a quantum system consisting of two nuclei (with charges $Z_{1}$ and $Z_{2}$ and masses $M_{1}$ and $M_{2}$ ) and $N$ electrons. The mass of an electron is +1 and the charge is -1 . The hamiltonian of the system $D_{1}$ in a homogeneous magnetic field has the form

$$
\begin{aligned}
\mathscr{H}= & \sum_{j=1}^{2} \frac{1}{2} M_{j}^{-1}\left(i \nabla_{j}+A_{j}\right)^{2}+\sum_{j=3}^{N+2} \frac{1}{2}\left(i \nabla_{j}+A_{j}\right)^{2}+Z_{1} Z_{2}\left|r_{12}\right|^{-1} \\
& -\sum_{j=1}^{2} \sum_{s=3}^{N+2} Z_{j}\left|r_{j s}\right|^{-1}+\sum_{s, t=3 ; s<t}^{N+2}\left|r_{s t}\right|^{-1}
\end{aligned}
$$

where $r_{j}=\left(r_{j}^{1}, r_{j}^{2}, r_{j}^{3}\right)$ are the coordinates of the $j^{\text {th }}$ particle,

$$
\begin{aligned}
& r_{j s}=\left(r_{s}-r_{j}\right), \quad A_{j}=\frac{B}{2} Z_{j}\left(r_{j}^{2},-r_{j}^{1}, 0\right) \quad j=1,2, \\
& A_{j}=\frac{B}{2} Z_{j}\left(-r_{j}^{2}, r_{j}^{1}, 0\right) \quad j \geqq 3 ; B>0 .
\end{aligned}
$$

The hamiltonian $\mathscr{H}$ does not contain a spin part because of reasons to be described later. We assume that $Z_{1}+Z_{2}>N$ (positive ions) and $Z_{2} \geqq Z_{1}$.

For $B=0$ one can separate the center of mass motion in the operator $\mathscr{H}$. The system $D_{1}$ is stable (for $B=0$ ) if the resulting operator $H_{0}$ has discrete spectrum on the subspace of functions with permutational symmetry satisfying the Pauli principle.

If $B \neq 0$ we can separate the center of mass motion only in the direction of the magnetic field (the $3^{\text {rd }}$ axis). So, for this case we need to modify the definition of stability.

Let us rewrite the operator $\mathscr{H}$ in the form

$$
\mathscr{H}=T+V,
$$


where $T$ is kinetic energy and $V$ is potential energy;

$$
\begin{aligned}
& T=\sum_{j=1}^{2} 1 / 2 M_{j}^{-1}\left(i \nabla_{j}+A_{j}\right)^{2}+\sum_{j=3}^{N+2} 1 / 2\left(i \nabla_{j}+A_{j}\right)^{2}, \\
& V=Z_{1} Z_{1}\left|r_{12}\right|^{-1}-\sum_{j=1}^{2} \sum_{s=3}^{N+2} Z_{j}\left|r_{j s}\right|^{-1}+\sum_{\substack{s, t=3 \\
s<t}}^{N+2} Z_{j}\left|r_{s t}\right|^{-1} .
\end{aligned}
$$

Operator $V$ depends only on the coordinates of the particles. Let

$$
\zeta_{c 3}=\left(\sum_{j=1}^{2} M_{j} r_{j}^{3}+\sum_{j=3}^{N+2} r_{j}^{3}\right) \cdot\left(\sum_{j=1}^{2} M_{j}+N\right)^{-1}
$$

be the $3^{\text {rd }}$ coordinate of the center of mass position vector of our system. Operator $T$ can be rewritten in the form

$$
T=T_{0}-1 / 2\left(M_{1}+M_{2}+N\right)^{-1} \frac{d^{2}}{d \zeta_{c 3}^{2}},
$$

where operator $T_{0}$ depends only on the coordinates in $R_{03}$

$$
R_{03}=\left\{r \mid r=\left(r_{1}, \ldots, r_{N+2}\right) \in R^{3(N+2)}, \sum_{j=1}^{3} M_{j} r_{j}^{3}+\sum_{j=3}^{N+2} r_{j}^{3}=0\right\}
$$

Operator

$$
-\frac{1}{2}\left(M_{1}+M_{2}+N\right)^{-1} \frac{d^{2}}{d \zeta_{c 3}^{2}}
$$

corresponds to the kinetic energy of the motion of the whole system in the direction of the magnetic field. Let

$$
\mathscr{H}_{0}=T_{0}+V .
$$

The stability of the positive molecular ion is determined by the spectral properties of the operator $\mathscr{H}_{0}$.

\section{3. $H V Z$ Theorem for the Operator $\mathscr{H}_{0}$ and the Definition of Stability}

Let $S$ be the group of permutations of identical particles in the system $D_{1}$ (electrons and also nuclei if they are identical), $\alpha$-be a type of irreducible representation of the group $S$.

The operator $\mathscr{H}_{0}$ is invariant under the actions of the group $G \equiv S \times S O(2) \times W$, where $S O(2)$ is a group of two-dimensional rotations around the direction of the $3^{\text {rd }}$ axis, $W$ is a group of reflections of the $3^{\text {rd }}$ axis.

Let $m$ be the weight of an irreducible representation of the $S O(2)$ group and $\omega= \pm 1$ is a parity. We shall denote by $\mathscr{H}_{0}^{\sigma}$ the restriction of the operator $\mathscr{H}_{0}$ onto the subspace of functions with symmetry $\sigma \equiv(\alpha, m, \omega)$. It is clear that $\mathscr{H}_{0}=$ $\sum_{\sigma} \bigoplus \mathscr{H}_{0}^{\sigma}$.

The important property of the operator $\mathscr{H}_{0}^{\sigma}$ is that the $H V Z$ theorem holds for it without any additional separation of the center of mass motion. 
The essential spectrum of $\mathscr{H}_{0}^{\sigma}$ is defined by decompositions of the system into clusters.

Another substitute for the separation of the center of mass motion for the operator $\mathscr{H}_{0}$ was suggested earlier by J. Avron, I. Herbst and B. Simon [1]. They proved that for the operator with fixed value of the pseudomomentum the HVZ theorem holds. From many points of view their approach is more general than one used in this paper. It works both in the neutral and charged cases. It is based on the translation invariance and does not need rotational symmetry of the potentials. Unfortunately operators with fixed pseudomomentum are more complicated for studying than those with fixed rotational symmetry. At the same time the whole set of operators $\mathscr{H}_{0}^{\sigma}$ for all $\sigma$ describes spectral properties of the original operator $\mathscr{H}_{0}$ and the considered physical system as well as the whole set of operators with fixed pseudomomentum.

To formulate the analogue of the HVZ theorem for our case we need to introduce some additional definitions.

Let $D_{2}=\left(C_{1}, C_{2}\right)$ be an arbitrary decomposition of the system into two noninteracting clusters,

$$
R_{0}\left[C_{j}\right]=\left\{r \mid r \in R_{03}, r_{i}=0, i \notin C_{j}\right\}, \quad R_{0}\left(D_{2}\right)=R_{0}\left[C_{1}\right] \oplus R_{0}\left[C_{2}\right] .
$$

For subsystems $C_{j}, j=1,2$ we define operators $\mathscr{H}_{0}\left[C_{j}\right]$ by the analogy with the operator $\mathscr{H}_{0}$ for the whole system.

The operator $\mathscr{H}_{0}\left[C_{j}\right]$ contains only variables corresponding to the particles in the subsystem $C_{j}$.

Let $\mathscr{H}_{0}\left(D_{2}\right)=\mathscr{H}_{0}\left[C_{1}\right]+\mathscr{H}_{0}\left[C_{2}\right]$. We denote by $S\left[C_{j}\right]$ a group of permutations of identical particles in $C_{j}$; $\hat{S}\left(D_{2}\right)$ be a group generated by $S\left[C_{1}\right] \times S\left[C_{2}\right]$ and a permutation of subsystems $C_{1} \leftrightarrow C_{2}$ if they are identical $\left(C_{1} \sim C_{2}\right)$.

Let $\hat{\alpha}, \hat{m}, \hat{\omega}$ be types of irreducible representations of the groups $\hat{S}\left(D_{2}\right), S O(2)$ and $W_{3}$ at $\mathscr{L}_{2}\left(R_{0}\left(D_{2}\right)\right)$. For $C_{1} \sim C_{2}$ by $\alpha_{c}^{-}$we denote a type of one dimensional irreducible representation of the group $\hat{S}\left(D_{2}\right)$ for which the number -1 corresponds to the permutation of clusters $C_{1}$ and $C_{2}$ and number +1 to all permutations from $S\left[C_{1}\right] \times S\left[C_{2}\right]$. Let $-\hat{\alpha}$ be the type of irreducible representation of the group $\hat{S}$ which is a direct product of the types $\hat{\alpha}$ and $\alpha_{c}^{-}$if $C_{1} \sim C_{2}$ and $-\hat{\alpha}=\hat{\alpha}$ if $C_{1} \psi C_{2}$. We shall write that the symmetry $\hat{\sigma}=(\hat{\alpha}, \hat{m}, \hat{\omega})$ is induced by the symmetry $\sigma=$ $(\alpha, m, \omega)(\hat{\sigma} \prec \sigma)$ if one of the next conditions holds

1) the representation $\hat{\alpha}$ is contained at the restriction of the representation $\alpha$ from $S$ to $\hat{S}\left(D_{2}\right), \hat{m}=m$ and $\hat{\omega}=\omega$;

2) the representation $-\hat{\alpha}$ is contained at the restriction of the representation $\alpha$ from $S$ to $\hat{S}\left(D_{2}\right), \hat{m}=m$ and $\hat{\omega}=-\omega$.

We denote by $P^{\hat{\sigma}}$ projector at $\mathscr{L}_{2}\left(R_{0}\left(D_{2}\right)\right)$ onto the subspace of functions with the symmetry $\hat{\sigma}$ and let

$$
\mathscr{H}_{0}^{\hat{\sigma}}\left(D_{2}\right)=\mathscr{H}_{0}\left(D_{2}\right) P^{\hat{\sigma}}, \quad \mathscr{H}_{0}\left(\sigma ; D_{2}\right)=\sum_{\hat{\sigma} \prec \sigma} \mathscr{H}_{0}^{\hat{\sigma}}\left(D_{2}\right), \quad \mu^{\sigma}=\min \inf \mathscr{H}_{0}\left(\sigma, D_{2}\right) .
$$

The location of the essential spectrum of the operator $\mathscr{H}_{0}^{\sigma}$ can be described by the next theorem of the HVZ type.

Theorem 1. The essential spectrum of the operator $\mathscr{H}_{0}^{\sigma}$ coincides with the half line $\left[\mu^{\sigma},+\infty\right)$. 
The proof of the theorem will be given in Appendix 1 .

The theorem shows that the bottom of the essential spectrum of the operator $\mathscr{H}_{0}^{\sigma}$ is defined by the decompositions of the system. So, bound states with the symmetry $\sigma$ correspond to the discrete spectrum of the operator $\mathscr{H}_{0}^{\sigma}$.

We shall study only the types of symmetry $\sigma$ for which permutational symmetry $\alpha$ satisfies the Pauli principle.

Definition 1. We shall say that a positive molecular ion is stable if there is at least one type of symmetry $\sigma$ satisfying the Pauli principle for which the discrete spectrum of $\mathscr{H}_{0}^{\sigma}$ is nonempty.

If for all physically realizable types $\sigma$ the discrete spectrum of the operator $\mathscr{H}_{0}^{\sigma}$ is empty, the positive molecular ion is unstable.

\section{Remarks.}

(1) Note that the discrete spectrum of one type of symmetry $\sigma_{1}$, may overlap the essential spectrum of the other type of symmetry $\sigma_{2}$. In this case a discrete eigenvalue of the operator $\mathscr{H}_{0}^{\sigma_{1}}$ corresponds to a stable state of the physical system. Our definition takes this situation into account.

(2) It is clear from the above definition that to study the stability of the positive molecular ion one should investigate the discrete spectrum of the operator $\mathscr{H}_{0}^{\sigma}$. If the type of the permutational symmetry $\alpha$ is fixed, the total spin of the electrons and spin of nuclei are fixed too. We can also consider the states with the fixed projection of the spin onto the direction of the magnetic field. For such subspaces the interaction term between spin and magnetic field in the hamiltonian gives only the shift of the spectrum. The existence of the bound states does not depend on it and this term may be omitted.

We shall give another definition of stability (which is equivalent to the first one due to Theorem 1) which makes clear that our first definition describes physical stability of the system.

Notice that the operator $\mathscr{H}$ is invariant under the actions of groups $S, S O(2)$ and $W_{3}$ and we can consider its restriction $\mathscr{H}^{\sigma}$ onto the subspace $\mathscr{B}^{\sigma}$ of functions with the symmetry $\sigma$. Let

$r=\left(r_{1}, \ldots, r_{N+2}\right) \in R^{3(N+2)}, \quad \rho(r)=\sum_{i, j=1}^{N+2}\left|r_{i j}\right|, \quad \Omega(a)=\left\{r \mid r \in R^{3(N+2)}, \rho(r) \geqq a\right\}$

and

$$
\mu_{1}^{\sigma}=\lim _{a \rightarrow \infty} \inf _{\substack{\psi \in \mathscr{g}^{\sigma} \\ \operatorname{supp}\{\psi \in \Omega(a)}}\left(\mathscr{H}^{\sigma} \psi, \psi\right) \cdot\|\psi\|^{-2} .
$$

Definition 2. We shall say that a molecular ion is stable if for at least one type of symmetry $\sigma$, satisfying the Pauli principle the next inequality holds

$$
\inf _{\psi \in \mathscr{B}^{\sigma}}\left(\mathscr{H}^{\sigma} \psi, \psi\right) \cdot\|\psi\|^{-2}<\mu_{1}^{\sigma} .
$$

By this definition the system is stable if at least for one type of symmetry, satisfying the Pauli principle, it has states with small distances among the particles for which the energy is less than the energy of decomposed system.

According to Theorem 1, this definition is equivalent to the first one and $\mu_{1}^{\sigma}=\mu^{\sigma}$. 


\section{Main Results}

Theorem 2. Let there be such a number $\delta_{0}>0$ that for $B, Z_{1}, Z_{2}$ the next inequality holds

$$
\left(B \cdot Z_{2}^{-2}\right)^{3 / 2}\left[\ln \left(1+B Z_{2}^{-2}\right)\right]^{3+\delta_{0}} \leqq Z_{1} .
$$

Then for any fixed $N$ there exists a constant $Z^{c}\left(\delta_{0}\right)$ such that if $Z_{1} \geqq Z^{c}$ the positive molecular ion is unstable, and for any fixed $\delta_{0}>0$

$$
\varlimsup_{N \rightarrow \infty} Z^{c} N^{-1} \leqq 2 \text {. }
$$

Remarks.

(1) Theorem 2 is a generalization of the results of [5] to the case $B>0$. Notice that our definition of stability does not work for the case $B=0$, but in this case the statement of Theorem 2 coincides with the statement of [5].

(2) Inequality (4.1) can be considered as a sufficient condition on magnetic field $B$ to get the same limits on stability as in [5].

(3) If magnetic field $B$ is not too strong $B \cdot Z_{2}^{-2} \leqq Z_{1}^{2 / 7}$ more precise estimates for the limit on stability can be given by the next statement.

Theorem 3. One can find such a constant $C_{0}>0$, that if one of the conditions 1,2 , or 3 holds, the positive molecular ion is unstable

(1) $\left(B Z_{2}^{-2}\right) \leqq Z_{1}^{-1 / 4}$ and $Z_{1} \geqq\left(2+C_{0} Z_{1}^{-1 / 12}\right) N$,

(2) $Z_{1}^{-1 / 4} \leqq\left(B Z_{2}^{-2}\right) \leqq 1$ and $Z_{1} \geqq\left(2+C_{0} Z_{1}^{-1 / 21}\left(B Z_{2}^{-2}\right)^{1 / 7}\right) N$,

(3) $1 \leqq\left(B Z_{2}^{-2}\right) \leqq Z_{1}^{2 / 7}$ and $Z_{1} \geqq\left(2+C_{0} Z_{1}^{-1 / 21}\left(B Z_{2}^{-2}\right)^{1 / 6}\right) N$.

Remark. Theorem 3 can not be applied directly to the case $B=0$, but it follows from the proof of this theorem that if $B=0$ and $Z_{1} \geqq\left(2+C_{0} Z_{1}^{-1 / 12}\right) N$ the positive molecular ion is unstable.

Consider now the case of strong magnetic field $\left(B Z_{2}^{-2}\right)^{3 / 2}\left[\ln \left(1+B Z_{2}^{-2}\right)\right]^{3} \geqq$ $Z_{1}$. In this situation an ability of the magnetic field to localize the system in two dimensions may be large in comparison with coulombic interaction between nuclei. So, for instability we should have nuclear charges many times greater than the number of electrons. Theorems 4 and 5 give conditions of instability for this case.

Theorem 4. Let the next inequality hold for some $\delta_{0} \in[0,5]$,

$$
\left(B Z_{2}^{-2}\right)^{3 / 2}\left[\ln \left(1+B Z_{2}^{-2}\right)\right]^{3-\delta_{0}} \leqq Z_{1} .
$$

Then one can find a number $C_{0}$ which does not depend on $Z_{1}, Z_{2}, N, \delta_{0}$ such that for

$$
Z_{1}>C_{0}\left[\ln \left(2+B Z_{2}^{-2}\right)\right]^{\frac{\delta_{0}}{3}} N
$$

the positive molecular ion is unstable.

Theorem 5. One can find a constant $C_{0}$ such that if the inequality

$$
Z_{1}>C_{0}\left[\ln \left(2+B Z_{2}^{-2}\right)\right] N
$$

holds the positive molecular ion is unstable. 


\section{Proof of Theorems 2-5}

5.1. To prove the theorems it suffices to demonstrate that under their conditions the inequality

$$
\left(\mathscr{H}_{0}^{\sigma} \psi, \psi\right) \geqq \mu^{\sigma}\|\psi\|^{2}
$$

holds for any $\psi \in C_{0}^{2}\left(R_{0}\right)$ such that $P^{\sigma} \psi=\psi$, where $P^{\sigma}$ is the projector in $\mathscr{L}_{2}\left(R^{3(N+2)}\right)$ onto the subspace of functions with the symmetry $\sigma$. Due to the inequality (2.5) relation (5.1) holds if and only if

$$
\left(\mathscr{H}^{\sigma} \psi, \psi\right) \geqq \mu^{\sigma}\|\psi\|^{2}
$$

for $\forall \psi \in C_{0}^{2}\left(R^{3(N+2)}\right), P^{\sigma} \psi=\psi$. Our goal is to verify the inequality (5.2).

5.2. Following the strategy of [5], let us separate two regions in the configuration space of the system: the large internuclear distance region and the small internuclear distance region. Estimates for the lower bound of the quadratic form of the hamiltonian are different in these regions.

Let $u(t), v(t) \in C^{2}\left(R_{+}^{1}\right), u^{2}+v^{2}=1, u(t)=1$ for $t \leqq 1, u(t)=0$ for $t \geqq 2$. It is obvious that

$$
\left(\mathscr{H}^{\sigma} \psi, \psi\right) \geqq L_{1}\left[\psi u\left(\left|r_{12}\right| \kappa^{-1} Z_{2}\right)\right]+L_{1}\left[\psi v\left(\left|r_{12}\right| \kappa^{-1} Z_{2}\right)\right]
$$

where

$$
L_{1}[\psi]=\left(\mathscr{H}^{\sigma} \psi, \psi\right)-c \kappa^{-2} Z_{2}^{2}\|\chi \psi\|^{2},
$$

$c$ depends only on $u(t)$ and $v(t), \chi$ - is a characteristic function of the region $\kappa Z_{2}^{-1} \leqq\left|r_{12}\right| \leqq 2 \kappa Z_{2}^{-1}, \kappa$ is parameter which will be chosen later. To prove the inequality (5.2) it suffices to show that

$$
L_{1}\left[\psi u\left(\left|r_{12}\right| \kappa^{-1} Z_{2}\right)\right] \geqq \mu^{\sigma}\|\psi\|^{2}
$$

and

$$
L_{1}\left[\psi v\left(\left|r_{12}\right| \kappa^{-1} Z_{2}\right)\right] \geqq \mu^{\sigma}\|\psi\|^{2} .
$$

Function $\psi u$ has a support in a region where the distance between the nuclei is small, function $\psi v$ has a support in the large internuclear distance region.

5.3. Large internuclear distance region (lower bound for $L_{1}[\psi v]$ ). Similar to the case $B=0$ [5] we shall decompose this region into subregions with the following properties:

(1) each subregion corresponds to a decomposition of the whole system into two subsystems $C_{1}$ and $C_{2}$ such that $C_{1}$ contains the first nucleus and $C_{2}$ contains the second one;

(2) the effective interaction between $C_{1}$ and $C_{2}$ is positive and large enough to compensate the localization error. 
Let $g(t) \in C^{\infty}\left(R_{+}^{1}\right)$ and (see [5])
a) $0 \leqq g(t) \leqq 1 \quad \forall t$,
b) $g(t)=0$ for $t \leqq(1+2 \varepsilon)$,
c) $g(t)=1$ for $t \leqq(1+2 \varepsilon)^{-1}$,
d) $g^{2}(t)+g^{2}\left(t^{-1}\right)=1 \quad \forall t$
e) $0 \leqq g^{\prime}(t) \leqq c_{0} \varepsilon^{-1}$,

where $c_{0}$-is a constant and a number $\varepsilon>0$ will be chosen later. For $i>2$ let $u_{1 i}=g\left(\left|r_{1 i}\right|\left|r_{2 i}\right|^{-1}\right), u_{2 i}=g\left(\left|r_{2 i}\right|\left|r_{1 i}\right|^{-1}\right)$. It is obvious that $u_{1 i}^{2}+u_{2 i}^{2}=1$.

Now we can define a partition of unity for the large internuclear distance region.

Let $D_{2}^{j}$ be an arbitrary decomposition of our system into nonempty subsystems $C_{1}$ and $C_{2}$ such that the particle 1 belongs to $C_{1}$ and the particle 2 belongs to $C_{2}$. For this decomposition we denote by $\mathscr{U}\left(D_{2}^{j}\right)$,

$$
\mathscr{U}\left(D_{2}^{j}\right)=\prod_{\substack{i \in C_{1} \\ t \neq 1}} u_{1 i} \prod_{\substack{p \in C_{2} \\ p \neq 2}} u_{2 p} .
$$

It is easy to see that

$$
\sum_{D_{2}^{j}} \mathscr{U}^{2}\left(D_{2}^{j}\right)=1
$$

and

$$
\begin{aligned}
L_{1}[\psi v]= & \sum_{D_{2}^{j}}\left\{L_{1}\left[\psi \mathscr{U}\left(D_{2}^{j}\right) v\right]-M_{1}^{-1}\left\|\left|\nabla_{1} \mathscr{U}\left(D_{2}^{j}\right)\right| \cdot \psi v\right\|^{2}\right. \\
& \left.-M_{2}^{-1}\left\|\left|\nabla_{2} \mathscr{U}\left(D_{2}^{j}\right)\right| \psi v\right\|^{2}-\sum_{p>2}\left\|\left|\nabla_{p} \mathscr{U}\left(D_{2}^{j}\right)\right| \cdot \psi v\right\|^{2}\right\} .
\end{aligned}
$$

The last three terms in the right-hand part of (5.7) are so-called "localization error." Let us estimate them.

It is obvious that for some constant $c_{1}>0$ and $p>2$,

$$
\left|\nabla_{p} \mathscr{U}\left(D_{2}^{j}\right)\right| \leqq c_{1} \varepsilon^{-2}\left|r_{12}\right|^{-2},
$$

and

$$
\begin{aligned}
\sum_{D_{2}^{j}}\left|\nabla_{1} \mathscr{U}\left(D_{2}^{j}\right)\right|^{2} & =\sum_{i>2}\left\{\left|\nabla_{1} u_{1 i}\right|^{2}+\left|\nabla_{1} u_{2 i}\right|^{2}\right\} \\
& \leqq N c_{2} \varepsilon^{-2}\left|r_{12}\right|^{-2}
\end{aligned}
$$

So, for some constant $c_{3}>0$,

$$
\begin{aligned}
& \sum_{D_{2}^{j}}\left\{M_{1}^{-1}\left\|\left|\nabla_{1} \mathscr{U}\left(D_{2}^{j}\right)\right| \psi v\right\|^{2}+M_{2}^{-1}\left\|\left|\nabla_{2} \mathscr{U}\left(D_{2}^{j}\right)\right| \psi v\right\|^{2}\right\}+\sum_{p}\left\|\left|\nabla_{p} \mathscr{U}\left(D_{2}^{j}\right)\right| \psi v\right\|^{2} \\
& \quad \leqq N c_{3} \varepsilon^{-2}\left\|\psi v\left|r_{12}\right|^{-1}\right\|^{2} .
\end{aligned}
$$

Taking into account that

$$
\sum_{D_{2}^{j}} \mathscr{U}^{2}\left(D_{2}^{j}\right)=1
$$


we get from (5.7) and (5.10) that

$$
L_{1}[\psi v] \geqq \sum_{D_{2}^{J}} L_{2}\left[\psi v \mathscr{U}\left(D_{2}^{j}\right)\right]
$$

where

$$
L_{2}[\varphi]=L_{1}[\varphi]-c_{3} N \varepsilon^{-2}\left\|\varphi\left|r_{12}\right|^{-1}\right\|^{2}
$$

5.4. Let us estimate now the lower bound of $L_{2}\left[\psi v \mathscr{U}\left(D_{2}^{j}\right)\right]$. Let $\psi_{1}=\psi v \mathscr{U}\left(D_{2}^{j}\right)$,

$$
\begin{aligned}
L_{2}\left[\psi_{1}\right] \geqq & \left(\mathscr{H}_{0}\left(D_{2}^{j}\right) \psi_{1}, \psi_{1}\right)+\left(\left\{-\sum_{p=1,2} Z_{p} \sum_{\substack{t \in c_{t} \\
t \neq p, l=1,2}}\left|r_{i j}\right|^{-1}\right.\right. \\
& \left.\left.+Z_{2} Z_{1}\left|r_{12}\right|^{-1}-\kappa^{-2} Z_{2}^{2} \chi-c_{3} N \varepsilon^{-2}\left|r_{12}\right|^{-2}\right\} \psi_{1}, \psi_{1}\right) .
\end{aligned}
$$

The function $\psi_{1}$ has the symmetry $\hat{\sigma}$ with respect to the actions of the group $\hat{S}\left(D_{2}\right) \times S O(2) \times W_{3}$ which is induced by the symmetry $\sigma(\hat{\sigma} \prec \sigma$ see Sect. 3$)$, so

$$
\left(\mathscr{H}_{0}\left(D_{2}^{j}\right) \psi_{1}, \psi_{1}\right) \geqq \mu^{\sigma}\|\psi\|^{2} .
$$

We shall prove that at the support of the function $\psi_{1}$ the next inequality holds:

$$
\begin{aligned}
I_{1} \equiv & -\sum_{p=1,2} Z_{p} \sum_{\substack{i \in c_{t} \\
t \neq p, i \neq 1,2}}\left|r_{i p}\right|^{-1}+Z_{1} Z_{2}\left|r_{12}\right|^{-1}-\kappa^{-2} Z_{2}^{2} \chi-c_{3} N \varepsilon^{-2}\left|r_{12}\right|^{-2} \\
& \geqq 0 .
\end{aligned}
$$

Notice that at the support of $\psi_{1}$ for $p=1,2 ; i \in C_{t}, t \neq p, i \neq p$,

$$
\left|r_{i p}\right|^{-1} \leqq 2(1+\varepsilon)\left|r_{12}\right|^{-1} \text {. }
$$

Hence

$$
\sum_{p=1,2} Z_{p} \sum_{\substack{i \in C_{t} \\ t \neq p, 1 \neq 1,2}}\left|r_{i p}\right|^{-1} \leqq 2(1+\varepsilon) Z_{2} N\left|r_{12}\right|^{-1}
$$

Moreover

$$
\chi \kappa^{-2} Z_{2}^{2} \leqq 2 \kappa^{-1} Z_{2}\left|r_{12}\right|
$$

so

$$
I_{1} \geqq\left|r_{12}\right|^{-1} Z_{2}\left\{-2(1+\varepsilon) N-2 \kappa^{-1}-c_{3} N \varepsilon^{-2} \kappa^{-1}+Z_{1}\right\} .
$$

Notice, that the inequality (5.16) holds for all $\varepsilon \in(0,1 / 4)$ and $\kappa>0$ and due to it and relation (5.7),

$$
L_{1}[\psi v] \geqq \mu^{\sigma}\|\psi v\|^{2}
$$

for all $\kappa>0$ and $\varepsilon \in(0,1 / 4)$ for which

$$
\left(-2(1+\varepsilon) N-2 \kappa^{-1}-c_{3} N \varepsilon^{-2} \kappa^{-1}+Z_{1}\right)>0 .
$$

5.5. Partition of unity for the small internuclear distance region and preliminary estimates. Let us estimate the lower bound of $L_{1}[\psi u]$. First of all, notice that

$$
\mu^{\sigma} \leqq \inf T \cdot P^{\sigma},
$$


where $P^{\sigma}$ is the projector onto the subspace of functions with the symmetry $\sigma$ and $T$ is the kinetic energy operator (see (2.2)). The inequality (5.18) follows from Theorem 1 if we consider the decomposition $D_{2}$ into subsystems $C_{1}$ and $C_{2}$ such that both nuclei are in the subsystem $C_{1}$ and all the electrons are in $C_{2}$. Obviously

$$
\inf T P^{\sigma}=\left(Z_{1} M_{1}^{-1}+Z_{2} M_{2}^{-1}+N\right) B \text {, }
$$

and hence

$$
\mu^{\sigma} \leqq\left(Z_{1} M_{1}^{-1}+Z_{2} M_{2}^{-1}+N\right) B
$$

5.6. Our next goal is to decompose the small internuclear distance region into subregions corresponding to different decompositions of the whole system into two clusters $\left(C_{1}^{i}, C_{2}^{i}\right)$ such that both nuclei are in one cluster $C_{1}^{i}$. In such subregions for electrons from the subsystem $C_{2}^{i}$ (inner electrons) the potential energy of the interaction with the nuclei is small relatively to the potential of the internuclear interaction. For the electrons from the subsystem $C_{1}^{i}$ it is not small, but all these electrons are localized in a small region of the configuration space and their kinetic energy is large.

Let $r_{0}=\left(r_{1} M_{1}+r_{2} M_{2}\right)\left(M_{1}+M_{2}\right)^{-1}$ be the position vector of the center of masses of nuclei, $r_{j 0}=r_{j}-r_{0}, u(t)$ and $v(t)$ are the same functions as in Sect. 5.2.

Denote

$$
u_{j 0}=u\left(\left|r_{j 0}\right| \kappa_{1}^{-1} \kappa^{-1} Z_{2}\right),
$$

where the number $\kappa_{1}$ will be chosen later. Let $v_{j 0}=\left(1-u_{j 0}^{2}\right)^{1 / 2}$.

For an arbitrary decomposition $D_{2}^{i}$ such that $C_{1}$ and $C_{2}$ are nonempty and both of the nuclei belong to $C_{1}$ define

$$
\begin{gathered}
U\left(D_{2}^{i}\right)=\prod_{\substack{j \in C_{1} \\
j>2}} u_{j 0} \prod_{p \in C_{2}} v_{p 0}, \\
U\left(D_{1}\right)=\prod_{j>2} u_{j 0}
\end{gathered}
$$

It is easy to see that

$$
\sum_{D_{2}^{l}} U^{2}\left(D_{2}^{i}\right)+U^{2}\left(D_{1}\right)=1
$$

and for $j>2$

$$
\sum_{D_{2}^{i}}\left|\nabla_{j} U\left(D_{2}^{i}\right)\right|^{2}+\left|\nabla_{j} U\left(D_{1}\right)\right|^{2} \leqq c \kappa_{1}^{-2} \kappa^{-2} Z_{2}^{2},
$$

where $c$ is a constant depending only on the function $u(t)$. Thus we have

$$
L_{1}[\psi u] \geqq L_{3}\left[\psi u U\left(D_{1}\right)\right]+\sum_{D_{2}^{i}} L_{3}\left[\psi u U\left(D_{2}^{i}\right)\right],
$$

where

$$
L_{3}[\varphi]=L_{1}[\varphi]-c \kappa_{1}^{-2} \kappa^{-2} Z_{2}^{2} N\|\varphi\|^{2} .
$$

Let us estimate $L_{3}\left[\psi u U\left(D_{2}^{i}\right)\right]$ for some fixed decomposition $D_{2}^{i}$. We will prove that

$$
L_{3}\left[\psi u U\left(D_{2}^{i}\right)\right] \geqq \mu^{\sigma}\left\|\psi u U\left(D_{2}^{i}\right)\right\|^{2} .
$$

The inequality

$$
L_{3}\left[\psi u U\left(D_{1}\right)\right] \geqq \mu^{\sigma}\left\|\psi u U\left(D_{1}\right)\right\|^{2}
$$

can be proved similar. 
5.7. Assume that $C_{1}$ contains electrons with the number $j=3, \ldots, p+2$. Let $\psi_{i}=\psi u U\left(D_{2}^{i}\right)$. Thus we have

$$
\begin{aligned}
L_{3}\left[\psi_{i}\right] \geqq & \left(T \psi_{i}, \psi_{i}\right)+Z_{1} Z_{2}\left(\left|r_{12}\right|^{-1} \psi_{i}, \psi_{i}\right)-\sum_{j=1}^{2} \sum_{s=3}^{N+2} Z_{j}\left(\left|r_{j s}\right|^{-1} \psi_{i}, \psi_{i}\right) \\
& -c \kappa^{-2} Z_{2}^{2}\left\|\psi_{i}\right\|^{2}-c \kappa_{1}^{-2} \kappa^{-2} Z_{2}^{2} N\left\|\psi_{i}\right\|^{2} .
\end{aligned}
$$

Notice, that at the support of the function $\psi_{i}$ for outer electrons $(s>p+2)$ and $j=1,2,\left|r_{j s}\right|>\left|r_{j 0}\right|-\left|r_{12}\right| \geqq \kappa\left(\kappa_{1}-2\right) Z_{2}^{-1}$ and $\left|r_{12}\right| \leqq 2 \kappa Z_{2}^{-1}$. Let $\kappa_{1}=34$, then

$$
\sum_{j=1}^{2} \sum_{s=p+3}^{N+2} Z_{j}\left(\left|r_{j s}\right|^{-1} \psi_{i}, \psi_{i}\right) \leqq 16^{-1} \kappa^{-1} N Z_{2}^{2}\left\|\psi_{i}\right\|^{2} \leqq 16^{-1} \kappa^{-1} Z_{1} Z_{2}^{2}\left\|\psi_{i}\right\|^{2} .
$$

On the other hand the lower bound for the positive term describing the internuclear interaction is

$$
Z_{1} Z_{2}\left(\left|r_{j s}\right|^{-1} \psi_{i}, \psi_{i}\right) \geqq 2^{-1} \kappa^{-1} Z_{1} Z_{2}^{2}\left\|\psi_{i}\right\|^{2}
$$

Let us pick $\kappa>\kappa_{0} \equiv 16 c$, then

$$
c \kappa^{-1}<16^{-1} Z_{1} \text { and } c \kappa_{1}^{-1} \kappa^{-1} N<16^{-1} Z_{1} \text {. }
$$

From the relations (5.26) to (5.28) it is clear that for $\kappa>\kappa_{0}$,

$$
L_{3}\left[\psi_{i}\right] \geqq\left(T \psi_{i}, \psi_{i}\right)+\frac{1}{4} Z_{1} Z_{2}^{2} \kappa^{-1}\left\|\psi_{i}\right\|^{2}-\sum_{j=1}^{2} \sum_{s=3}^{p+2} Z_{j}\left(\left|r_{j s}\right|^{-1} \psi_{i}, \psi_{i}\right) .
$$

Summarizing the results of Sects. 5.1-5.7 we can say that to prove Theorem 3 it suffices to show that the parameters $\varepsilon \in(0,1 / 4)$ and $\kappa>\kappa_{0}$ may be chosen such that (5.17) holds and at the same time

$$
\left(T \psi_{i}, \psi_{i}\right)+\frac{1}{4} Z_{1} Z_{2}^{2} \kappa^{-1}\left\|\psi_{i}\right\|^{2}-\sum_{j=1}^{2} \sum_{s=3}^{p+2} Z_{j}\left(\left|r_{j s}\right|^{-1} \psi_{i}, \psi_{i}\right)>\mu^{\sigma}\left\|\psi_{i}\right\|^{2},
$$

where $\psi_{i}$ is the function described above.

5.8. Proof of Theorem 3. Now we are going to use our preliminary estimates for proving the theorems. Notice that for $B Z_{2}^{-2} \leqq Z_{1}^{2 / 7}$ the statement of Theorem 2 follows from Theorem 3 . Let us start from this case.

Let us pick $\varepsilon=Z_{1}^{-1 / 12}$ if condition 1 of Theorem 3 holds, $\varepsilon=Z_{1}^{-1 / 21}\left(B Z_{2}^{-2}\right)^{1 / 7}$ if condition 2 holds and $\varepsilon=Z_{1}^{-1 / 21}\left(B Z_{2}^{-2}\right)^{1 / 6}$ if condition 3 holds; $\kappa=\varepsilon_{0} \cdot \varepsilon^{-3}$. The parameter $\varepsilon_{0}>0$ will be chosen later (see Sect. 5.11). Notice that the lower bound of $\varepsilon_{0}$ is defined by the condition $\kappa>\kappa_{0}$.

It is clear that for any fixed $\varepsilon_{0}$ we can take $C_{0}$ at the conditions of the theorem such that the inequality $(5.17)$ holds. So, we should verify only the inequality (5.30) for $\kappa>\kappa_{0}$.

5.9. Let $\delta \in(0,1]$ and $\delta_{1} \in(0,1]$ be some positive numbers, which will be chosen later,

$$
T=T-\delta \sum_{j=3}^{p+2}\left(i \nabla_{j}+A_{j}\right)^{2}+\delta \delta_{1} \sum_{s=3}^{p+2}\left(i \nabla_{j}+A_{j}\right)^{2}+\delta\left(1-\delta_{1}\right) \sum_{j=3}^{p+2}\left(i \nabla_{j}+A_{j}\right)^{2}
$$


It is obvious that

$$
\begin{aligned}
\left(\left[T-\delta \sum_{j=3}^{p+2}\left(i \nabla_{j}+A_{j}\right)^{2}\right] \psi_{i}, \psi_{i}\right) & \geqq\left[M_{1} Z_{1}^{-1}+M_{2} Z_{2}^{-1}+(1-\delta) p+(N-p)\right] B\left\|\psi_{i}\right\|^{2} \\
& \geqq\left(\mu^{\sigma}-\delta B p\right)\left\|\psi_{i}\right\|^{2}
\end{aligned}
$$

By the corollary of the diamagnetic inequality [2]

$$
\left(\left(i \nabla_{j}+A_{j}\right)^{2} \psi_{i}, \psi_{i}\right) \geqq\left\|\nabla_{j}\left|\psi_{i}\right|\right\|^{2} .
$$

Thus for $t=1,2$ we have

$$
\begin{aligned}
\inf \left\{\frac{1}{2} \delta \delta_{1}\left(i \nabla_{j}+A_{j}\right)^{2}-Z_{t}\left|r_{j t}\right|^{-1}\right\} & \geqq \inf \left\{-\frac{1}{2} \delta \delta_{1} \Delta_{r_{j t}}-Z_{t}\left|r_{j t}\right|^{-1}\right\} \\
& =-\frac{1}{2} \delta^{-1} \delta_{1}^{-1} Z_{t}^{2} \geqq-\frac{1}{2} \delta^{-1} \delta_{1}^{-1} Z_{2}^{2} .
\end{aligned}
$$

It follows from the relations $(5.30)-(5.33)$, that

$$
\begin{aligned}
L_{3}\left[\psi_{i}\right] \geqq & \left.\mu^{\sigma}\left\|\psi_{i}\right\|^{2}-\delta B p\left\|\psi_{i}\right\|^{2}+\delta\left(1-\delta_{1}\right) \sum_{j=3}^{p+2}\left(\left(i \nabla_{j}+A_{j}\right)^{2}\right) \psi_{i} \psi_{i}\right) \\
& \times 4^{-1} Z_{1} Z_{2}^{2} \kappa^{-1}\left\|\psi_{i}\right\|^{2}-p \delta^{-1} \delta_{1}^{-1} Z_{2}^{2}\left\|\psi_{i}\right\|^{2}
\end{aligned}
$$

5.10. Let us consider now a subsystem $\bar{C}_{1}$ consisting of electrons with the numbers $j=3, \ldots, p+2$. The subsystem $\bar{C}_{1}$ is localized in a small region of the configuration space. The term $\left.\sum_{j=3}^{p+2}\left(\left(i \nabla_{j}+A_{j}\right)^{2}\right) \psi_{i}, \psi_{i}\right)$ corresponds to the kinetic energy $\bar{C}_{1}$. To estimate it, notice that all the particles in $\bar{C}_{1}$ are identical and we can separate its center of mass motion. Define

$$
\begin{aligned}
\zeta & =\left(\zeta^{(1)} \zeta^{(2)} \zeta^{(3)}\right)=p^{-1} \sum_{j=3}^{p+2} r_{j}, \\
r_{j 0} & =\left(r_{j 0}^{(1)} r_{j 0}^{(2)} r_{j 0}^{(3)}\right)=r_{j}-\zeta, \\
\nabla_{j 0} & =\nabla_{j}-p^{-1} \sum_{t=3}^{p+2} \nabla_{t},
\end{aligned}
$$

then

$$
\sum_{j=3}^{p+2}\left(i \nabla_{j}+A_{j}\right)^{2}=\left(i \nabla_{\zeta}+A_{\zeta}\right)^{2}+\sum_{j=3}^{p+2}\left(i \nabla_{j 0}+A_{j 0}\right)^{2} \geqq \sum_{j=3}^{p+2}\left(i \nabla_{j 0}+A_{j 0}\right)^{2},
$$

where

$$
\begin{aligned}
& A_{j 0}=\left\{-\frac{B}{2} r_{j 0}^{2}, \frac{B}{2} r_{j 0}^{1}, 0\right\}, \\
& A_{\zeta}=\left\{-\frac{B}{2} \zeta^{2}, \frac{B}{2} \zeta^{1}, 0\right\} .
\end{aligned}
$$

Let us estimate the right-hand part of (5.35). Obviously

$$
\left(\left(i \nabla_{j 0}+A_{j 0}\right)^{2} \psi_{i}, \psi_{i}\right) \geqq \frac{1}{2}\left\|\nabla_{j 0} \psi_{i}\right\|^{2}-\left\|\left|A_{j 0}\right| \psi_{i}\right\|^{2} .
$$


At the support of $\psi_{i}$,

$$
\left|A_{j 0}\right| \leqq 2 B \kappa_{1} \kappa Z_{2}^{-1}
$$

Thus we have

$$
\begin{aligned}
\sum_{j=3}^{p+2}\left(\left(i \nabla_{j 0}+A_{j 0}\right)^{2} \psi_{i}, \psi_{i}\right) & \geqq \frac{1}{2} \sum_{j=3}^{p+2}\left\|\nabla_{j 0} \psi_{i}\right\|^{2}-4 p B^{2} \kappa_{1}^{2} \kappa^{2} Z_{2}^{-2}\left\|\psi_{i}\right\|^{2} \\
& =-\left(\Delta_{0} \psi_{i}, \psi_{i}\right)-4 p B^{2} \kappa_{1}^{2} \kappa^{2} Z_{2}^{-2}\left\|\psi_{i}\right\|^{2},
\end{aligned}
$$

where $\Delta_{0}$ is Laplacian on the subspace $R_{0}$ of the relative motion of the subsystem $\bar{C}_{1}$,

$$
R_{0}=\left\{r \mid r=\left(r_{3}, \ldots, r_{p+2}\right) \in R^{3 p}, \sum_{j=3}^{p+2} r_{j}=0\right\}
$$

The function $\psi_{i}$ as a function of $r_{3}, \ldots, r_{p+2}$ has a symmetry satisfying the Pauli principle and according to Lemma A2.1 (Appendix 2) for sufficiently small $\gamma>0$,

$$
-\left(\Delta_{0} \psi_{i}, \psi_{i}\right) \geqq \gamma p^{5 / 3} \kappa_{1}^{-2} \kappa^{-2} Z_{2}^{2}\left\|\psi_{i}\right\|^{2} .
$$

By the relations (5.34)-(5.38),

$$
\begin{aligned}
L_{3}\left[\psi_{i}\right] \geqq & \mu^{\sigma}\left\|\psi_{i}\right\|^{2}+\left\{\gamma p^{5 / 3} \kappa_{1}^{-2} \kappa^{-2} \delta\left(1-\delta_{1}\right) Z_{2}^{2}+4^{-1} Z_{1} Z_{2}^{2} \kappa^{-1}\right. \\
& \left.-\delta B p-4 \delta\left(1-\delta_{1}\right) p B^{2} \kappa_{1}^{2} \kappa^{2} Z_{2}^{-2}-p \delta^{-1} \delta_{1}^{-1} Z_{2}^{2}\right\}\left\|\psi_{i}\right\|^{2} .
\end{aligned}
$$

5.11. It follows from (5.39) that to prove the theorem it suffices to show that under its conditions for $\kappa_{1}=34$ and $\kappa>\kappa_{0}$ the next inequality holds

$$
\begin{gathered}
\delta\left(1-\delta_{1}\right) \gamma p^{5 / 3} \kappa_{1}^{-2} \kappa^{-2} Z_{2}^{2}+4^{-1} Z_{1} Z_{2}^{2} \kappa^{-1}-\delta B p \\
-4 \delta\left(1-\delta_{1}\right) p B^{2} \kappa_{1}^{2} \kappa^{2} Z_{2}^{-2}-p \delta^{-1} \delta_{1}^{-1} Z_{2}^{2} \geqq 0 .
\end{gathered}
$$

First, notice that if the number of electrons $p$ in $\bar{C}_{1}$ is small $\left(p \ll Z_{1}\right)$ inequality (5.40) should hold, because the largest term in this case is the term $4^{-1} Z_{1} Z_{2}^{2} \kappa^{-1}$. At the support of the function $\psi_{i}$ the internuclear distance is less than $2 \kappa^{-1}$ and this term corresponds to $1 / 2$ of the potential energy of interaction between the nuclei.

On the other hand, if $p$ is very large the kinetic energy of electrons increases as $p^{5 / 3}$ and all negative terms as $p$. So in this case the inequality (5.40) holds too.

Let $B Z_{2}^{-2} \leqq Z_{1}^{-1 / 4}$ (condition 1 of the theorem holds). In this case $\kappa=\varepsilon_{0} Z_{1}^{1 / 4}$. We pick $\delta=1 / 2, \delta_{1}=1 / 2, p_{0}=\varepsilon_{0}^{3 / 5} Z_{1}^{3 / 4}$. Suppose for a moment that we can pick $\varepsilon_{0}$ sufficiently small. Then for $p \leqq p_{0}$,

$$
4^{-1} Z_{1} Z_{2}^{2} \kappa^{-2} \geqq \delta B p+4 \delta\left(1-\delta_{1}\right) p B^{2} \kappa_{1}^{2} \kappa^{2} Z_{2}^{2}+p \delta^{-1} \delta_{1}^{-1} Z_{2}^{2},
$$

and for $p>p_{0}$

$$
\gamma p^{5 / 3} \kappa_{1}^{-2} \kappa^{-2} Z_{2}^{2} \delta\left(1-\delta_{1}\right) \geqq \delta B p+4 \delta\left(1-\delta_{1}\right) p B^{2} \kappa_{1}^{2} \kappa^{2} Z_{2}^{2}+p \delta^{-1} \delta_{1}^{-1} Z_{2}^{2} .
$$


Let $\bar{\varepsilon}_{0}$ be a value of $\varepsilon_{0}$ such that for $\varepsilon_{0}=\bar{\varepsilon}_{0}$ the inequalities (5.41) and (5.42) hold for $p \leqq p_{0}$ and $p>p_{0}$ respectively. Recall that $\kappa=\varepsilon_{0} Z_{1}^{1 / 4}$ and to satisfy the condition $\kappa>\kappa_{0}$ we must pick $\varepsilon_{0}>\kappa_{0} Z_{1}^{-1 / 4}$. Let us show that if the number $C_{0}$ in the theorem is greater than $\kappa_{0}^{13 / 3} \bar{\varepsilon}_{0}^{-13 / 3}$, the minimal value of the charge $Z_{1}$, satisfying the condition $Z_{1} \geqq\left(2+C_{0} Z_{1}^{-1 / 12}\right) N$ is large and $\bar{\varepsilon}_{0}>\kappa Z_{1}^{-1 / 4}$. Really, for all $N \geqq 1$ we have $Z_{1} \geqq C_{0}^{12 / 13} N^{12 / 13}>\kappa_{0}^{4} \bar{\varepsilon}_{0}{ }^{4}$. Consequently $\kappa=\bar{\varepsilon}_{0} Z_{1}^{1 / 4}>\kappa_{0}$. Hence both the inequalities (5.40) and $\kappa>\kappa_{0}$ hold, which completes the proof of the theorem for $B Z_{2}^{-2} \leqq Z_{1}^{-1 / 4}$.

Let us proceed to the case $Z_{1}^{-1 / 4} \leqq\left(B Z_{2}^{-2}\right) \leqq 1$. For this case we pick $\delta=1 / 2$, $\delta_{1}=\left(1-\delta_{2}\right)$, where $\delta_{2}=\min \left\{1 / 2,\left(B Z_{2}^{-2}\right)^{-2} \kappa^{-2}\right\}, \kappa=\varepsilon_{0} Z_{1}^{1 / 7}\left(B Z_{2}^{-2}\right)^{-3 / 7} p_{0}=\left(Z_{1}\right.$ $\left.\kappa\left(1-\delta_{1}\right)^{-1}\right)^{3 / 5}$. Then the inequalities $(5.41),(5.42)$ hold for $\varepsilon_{0}$ small enough. Similar to the case $B Z_{2}^{-2} \leqq Z_{1}^{-1 / 4}$ for any fixed $\varepsilon_{0}$ we can find such a large number $C_{0}$ that the inequality $\kappa>\kappa_{0}$ is fulfilled, which completes the proof for $B Z_{2}^{-2} \leqq 1$.

If $1 \leqq\left(B Z_{2}^{-2}\right) \leqq Z_{1}^{2 / 7}$ and condition 3 of the theorem holds, the inequalities (5.41), (5.42) are fulfilled for $\delta_{1}=\left(1-\delta_{2}\right), \delta_{2}=\min \left\{1 / 2,\left(B Z_{2}^{-2}\right)^{-1} \kappa^{-2}\right\}, \delta=$ $1 / 2\left(B Z_{2}^{-2}\right)^{-1 / 2} p_{0}=Z_{1}^{3 / 5} \kappa^{9 / 5}\left(B Z_{2}^{-2}\right)^{9 / 10}$ and $\kappa=\varepsilon_{0} Z_{1}^{1 / 7}\left(B Z_{2}^{-2}\right)^{-1 / 2}$. The theorem is proved.

5.12. Proof of Theorem 2. To prove the theorem it suffices to consider only the strong magnetic field case $\left(B Z_{2}^{-2} \geqq Z_{1}^{2 / 7}\right)$. Let $\varepsilon_{2}>0$ be a fixed number, $Z_{1}>$ $\left(2+\varepsilon_{2}\right) N$. We should show that if $N$ is large enough the inequality (5.1) holds. Let us pick $\varepsilon=4^{-1} \varepsilon_{2}$ and $\kappa>\max \left\{c_{3}^{-1} \varepsilon^{-3}, \kappa_{0}\right\}$. According to (5.16) we have

$$
I_{1} \geqq\left|r_{12}\right| Z_{2}\left\{4^{-1} \varepsilon_{2} N-2^{-5} c_{3} \varepsilon_{2}\right\}>0
$$

for sufficiently large $N$, depending on $\varepsilon_{2}$.

Recall that the function $I_{1}$ was defined in Sect. 5.4. It follows from the above inequality that $L_{1}[\psi v] \geqq \mu^{\sigma}\|\psi v\|^{2}$.

The strategy of estimating of the lower bound for $L_{3}[\psi u]$ is the same as in the case of weak magnetic field (see Sect. 5.11). We shall consider separately two different cases: when $p$ (the number of electrons in $\bar{C}_{1}$ ) is large and when it is small. The difference between the strong magnetic field case and the weak one is in another estimate for kinetic energy of the electrons (see Appendix 3) and another lower bound of the energy of Hydrogen atom (see (5.44)).

Let $\delta=1 / 2, \delta_{1}=1$. According to (5.31), (5.32),

$$
\left(T \psi_{i}, \psi_{i}\right) \geqq \mu^{\sigma}\left\|\psi_{i}\right\|^{2}-1 / 2 B p\left\|\psi_{i}\right\|^{2}+1 / 2 \sum_{j=3}^{p+2}\left(\left(i \nabla_{j}+A_{j}\right)^{2} \psi_{i}, \psi_{i}\right) .
$$

Let us consider the operator

$$
h=\left(i \nabla_{j}+A_{j}\right)^{2}-Z_{t}\left|r_{j t}\right|^{-1}
$$

in $L_{2}\left(R^{3}\right)$ for $t=1,2, r_{t}$ is fixed. It is unitary equivalent to the operator.

$$
h_{1}=\left(i \nabla_{j}+A_{j}\right)^{2}-Z_{t}\left|r_{j}\right|^{-1} \text {. }
$$


Recall that we consider the case when $N \rightarrow \infty, Z_{1} \rightarrow \infty$ and $B Z_{2}^{-2} \geqq Z_{1}^{2 / 7} \rightarrow \infty$. According to [1] for large $B Z_{t}^{-2}$,

$$
\inf h=\inf h_{1} \geqq B-\frac{1}{2} Z_{t}^{2} \ln ^{2}\left(B Z_{t}^{-2}\right) .
$$

Thus we have

$$
\frac{1}{4} \sum_{j=3}^{p+2}\left(i \nabla_{j}+A_{j}\right)^{2}-\sum_{t=1,2} \sum_{j=3}^{p+2} Z_{t}\left|r_{t j}\right|^{-1} \geqq 4^{-1} B p-8 p Z_{2}^{2} \ln ^{2} B Z^{-2} .
$$

From the relations $(5.43),(5.45)$ and $(5.30)$ it follows that

$$
\begin{aligned}
L_{3}\left[\psi_{i}\right] \geqq & \mu^{\sigma}\left\|\psi_{i}\right\|^{2}+4^{-1} \sum_{j=3}^{p+2}\left(\left(i \nabla_{j}+A_{j}\right)^{2} \psi_{i}, \psi_{i}\right) \\
& +4^{-1} Z_{1} Z_{2}^{2} \kappa^{-1}\left\|\psi_{i}\right\|^{2}-4^{-1} B p\left\|\psi_{i}\right\|^{2}-8 p Z_{2}^{2} \ln ^{2}\left(B Z_{2}^{-2}\right)\left\|\psi_{i}\right\|^{2} .
\end{aligned}
$$

Let $\kappa>\kappa_{0}$ be fixed. For $p<2^{-5} \kappa^{-1} Z_{1} \ln ^{-2}\left(B Z_{2}^{-2}\right)$ the lower bound $L_{3}\left[\psi_{i}\right] \geqq$ $\mu^{\sigma}\left\|\psi_{i}\right\|$ holds, because $\inf \left(i \nabla_{j}+A_{j}\right)^{2}=B$. Thus it suffices to consider only the case $p \geqq 2^{-5} \kappa^{-1} Z_{1} \ln ^{-2}\left(B Z_{2}^{-2}\right)$. that

According to Theorem A3 (see Appendix 3), there is such a constant $\gamma_{2}>0$,

$$
\sum_{j=3}^{p+2}\left(\left(i \nabla_{j}+A_{j}\right)^{2} \psi_{i}, \psi_{i}\right) \geqq \min \left\{2 B p, B p+\gamma_{2} p^{3} B^{-3} \kappa_{1}^{-8} \cdot \kappa^{-8} Z_{2}^{8}\right\}\left\|\psi_{i}\right\|^{2} .
$$

If $B p>\gamma_{2} p^{8} B^{-3} \kappa_{1}^{-8} \cdot \kappa^{-8} Z_{2}^{8}$ it follows from (5.46) and (5.47) that

$$
L_{3}\left[\psi_{i}\right] \geqq \mu^{\sigma}\left\|\psi_{i}\right\|^{2}+4^{-1} B p\left\|\psi_{i}\right\|^{2}-8 p Z_{2}^{2} \ln ^{2}\left(B Z_{2}^{-2}\right) \cdot\left\|\psi_{i}\right\|^{2} .
$$

Recall that $B Z_{2}^{-2} \geqq Z_{1}^{2 / 7}$ and $Z_{1} \rightarrow \infty$. Consequently $B>32 Z_{2}^{2} \ln ^{2}\left(B Z_{2}^{-2}\right)$ and $L_{3}\left[\psi_{i}\right] \geqq \mu^{\sigma}\left\|\psi_{i}\right\|^{2}$. (5.47),

Let us assume that $B p<\gamma_{2} p^{3} B^{-3} \kappa_{1}^{-8} \cdot \kappa^{-8} Z_{2}^{8}$. By the inequalities (5.46) and

$$
\begin{aligned}
L_{3}\left[\psi_{i}\right] & \geqq \mu^{\sigma}\left\|\psi_{i}\right\|^{2}+\left\{\gamma_{2} p^{3} B^{-3} \kappa_{1}^{-8} \cdot \kappa^{-8} Z_{2}^{8}-8 p Z_{2}^{2} \ln ^{2}\left(B Z_{2}^{-2}\right)\right\} \cdot\left\|\psi_{i}\right\|^{2} \\
& \geqq \mu^{\sigma}\left\|\psi_{i}\right\|^{2}
\end{aligned}
$$

for large $N$, because $\kappa_{1}$ and $\kappa$ are fixed, $p \geqq$ const $\cdot Z_{1} \ln ^{-2}\left(B Z_{2}^{-2}\right), Z_{1}>N$ and $\left(B \cdot Z_{2}^{-2}\right)^{3 / 2}\left[\ln \left(B Z_{2}^{-2}\right)\right]^{3+\delta_{0}}<Z_{1}$. Theorem 2 is proved.

5.13. Proof of Theorems 4 and 5. The main ideas of the proof are the same as in Theorems 2 and 3, but in Theorems 2 and 3 we made the small internuclear distance region large relative to $Z_{2}^{-2}$. In Theorems 4 and $5 Z_{1} / N \gg 1$ and to get the optimal bound we pick the large internuclear distance region greater.

Let us take in (5.17), $\varepsilon=4^{-1}, C_{0}>2^{5}\left(c_{3}+4\right) \varepsilon_{2}^{-1}, \kappa=\varepsilon_{2}\left[\ln B Z_{2}^{-2}\right]^{-\delta_{0} / 5}$ for Theorem 4 and $\kappa=\varepsilon_{2}\left[\ln B Z_{2}^{-2}\right]^{-1}$ for Theorem 5 . The number $\varepsilon_{2}$ will be chosen later.

In this case the inequality (5.17) holds and it suffices to verify only (5.30) and the inequalities (see Sect. 5.7)

$$
c \kappa^{-1}<Z_{1} \text { and } c \kappa_{1}^{-1} \kappa^{-1} N<16^{-1} Z_{1} \text {. }
$$


If the conditions of Theorem 5 hold $Z_{1}>C_{0}\left(\ln B Z_{2}^{-2}\right) N$ and $\kappa=\varepsilon_{2}\left(\ln B Z_{2}^{-2}\right)^{-1}$. Hence for fixed $\varepsilon_{2}>0, \kappa_{1}>0, c>0$, we can choose $C_{0}$ such that (5.49) holds. Let us check (5.30). As in the proof of Theorem 2 we have inequality (5.45). For small $\varepsilon_{2}>0$,

$$
4^{-1} Z_{1} Z_{2}^{2} \kappa^{-1}>4^{-1} C_{0}\left(\ln B Z_{2}^{-2}\right)^{2} Z_{2}^{2} N \varepsilon_{2}^{-1}>8 N Z_{2}^{2} \ln ^{2}\left(B Z_{2}^{-2}\right)^{2} .
$$

It follows from (5.50) and (5.45), that

$$
\begin{aligned}
& \left(T \psi_{i}, \psi_{i}\right)+\frac{1}{4} Z_{1} Z_{2}^{-2} \kappa^{-1}\left\|\psi_{i}\right\|^{2}-\sum_{j=1}^{2} \sum_{S=3}^{p+2} Z_{j}\left(\left|r_{j s}\right|^{-1} \psi_{i}, \psi_{i}\right) \\
& \geqq 4^{-1}\left\{\sum_{j=3}^{p+2}\left(\left(i \nabla_{j}+A_{j}\right)^{2} \psi_{i}, \psi_{i}\right)-B p\left\|\psi_{i}\right\|^{2}\right\}+\mu^{\sigma}\left\|\psi_{i}\right\|^{2} \geqq \mu^{\sigma}\left\|\psi_{i}\right\|^{2} .
\end{aligned}
$$

Theorem 5 is proved.

Let us assume now that the conditions of Theorem 4 hold. In this case $Z_{1}>C_{0}\left[\ln B Z_{2}^{-2}\right]^{\delta_{0} / 5} N, \quad \kappa=\varepsilon_{2}\left[\ln B Z_{2}^{-2}\right]^{-\delta_{0} / 5}$. As in the proof of Theorem 2 the inequality (5.30) holds for $p<2^{-5} \kappa^{-1} Z_{1} \ln ^{-2}\left(B Z_{2}^{-2}\right)$ and it suffices to consider only the case $p \geqq 2^{-5} \kappa^{-1} Z_{1} \ln ^{-2}\left(B Z_{2}^{-2}\right)$. For this case (5.48) holds and it is easy to see that under the conditions of the theorem

$$
\gamma_{2} p^{3} B^{-3} \kappa_{1}^{-8} \cdot \kappa^{-8} Z_{2}^{8}-8 p Z_{2}^{2}\left[\ln ^{-2}\left(B Z_{2}^{-2}\right)\right]^{2}>0
$$

for fixed $\kappa_{1}$ and sufficiently small $\varepsilon_{2}$. Hence for small $\varepsilon_{2}>0$ (5.48) implies (5.30). Moreover, for chosen $\varepsilon_{2}>0$ and fixed $c$ and $\kappa_{1}$ we can pick $C_{0}$ such that the inequality (5.49) holds also, which completes the proof of Theorem 4.

\section{Appendix 1.}

Proof of Theorem 1. To prove the theorem it suffices to show that:

1. any $\lambda \in\left[\mu^{\sigma},+\infty\right)$ is a point of essential spectrum of the operator $\mathscr{H}_{0}^{\sigma}$;

2. there is no $\lambda<\mu^{\sigma}$ such that $\lambda$ is a point of the essential spectrum.

The first statement can be proved by analogy with the $H V Z$ theorem in the case $B=0[3,7]$.

The main idea of the proof of the second one is also the same as in the case $B=0$, but for $B \neq 0$ we separate the center of mass motion and for any fixed $A$, the region in the configuration space where all the distances between the particles are less than $A$ is compact. Because of this compactness for any fixed level of energy only a finite number of orthogonal states with the energy less than this level supported in this region may exist.

In the case $B \neq 0$ we separate the center of mass motion only in the direction of the magnetic field and the obtained region is not compact. Nevertheless the following important lemma holds. 
Lemma A1.1. Let $T_{0}^{\sigma}=T_{0} P^{\sigma}$ be the restriction of the operator $T_{0}$ onto the subspace of functions with the symmetry $\sigma=(\alpha, m, \omega), \chi_{a}(r) \in C_{0}^{\infty}\left(R_{03}\right), \chi_{a}(r)=1$ if $\left|r_{i}-r_{j}\right| \leqq a$ for $i, j=1,2, \ldots, N+2, \chi_{a}(r)=0$ if $\exists(i, j)$ that $\left|r_{i}-r_{j}\right| \geqq 2 a$. Then for any $c>0$ and $a>0$ one can find a finite dimensional subspace $M$ such that for any $\varphi \in P^{\sigma} \mathscr{L}_{2}\left(R_{03}\right), \varphi \perp M$ the next inequality holds

$$
\left(\chi_{a} T_{0}^{\sigma} \chi_{a} \varphi, \varphi\right) \geqq c\left\|\chi_{a} \varphi\right\|^{2} \text {. }
$$

Proof. By analogy with $\chi_{a}(r)$ let us define functions $\chi_{a, 3}(r)$ and $\chi_{a, \rho}(r)$. For any $r_{i}=\left(r_{i}^{1}, r_{i}^{2}, r_{i}^{3}\right)$, let $\rho_{i}=\left[\left(r_{i}^{1}\right)^{2}+\left(r_{i}^{2}\right)^{2}\right]^{1 / 2}$. We shall use for $\chi_{a, 3}$ and $\chi_{a, \rho}$ the same definition as for $\chi_{a}$ but in it we replace $r_{i}$ by $r_{i}^{3}, i=1, \ldots, N+2$ to obtain the function $\chi_{a, 3}$ and $r_{i}$ by $\rho_{i}, i=1,2, \ldots, N+2$ to obtain the function $\chi_{a, \rho}$.

Let $\hat{m}=\left(m_{1}, \ldots, m_{N+2}\right)$ and $P^{\hat{m}}$ be the projector onto the subspace of functions possessing symmetry of the weight $\hat{m}=\left(m_{1}, \ldots, m_{N+2}\right)$ with respect to the group

$$
\underbrace{S O(2) \times \cdots \times S O(2)}_{(N+2) \text { times }}, \quad T_{0}^{\hat{m}}=T_{0} P^{\hat{m}} .
$$

It is easy to see, that for any multi-index $\hat{m}$ the operator $\chi_{a, 3} T_{0}^{\hat{m}} \chi_{a, 3}$ has pure discrete spectrum. So, to prove the lemma we can show that there is only a finite number of multi-indexes $\hat{m}=\left(m_{1}, \ldots, m_{N+2}\right)$ for which $\sum_{i=1}^{N+2} m_{i}=m$ and

$$
\left(\chi_{2 a, \rho} T_{0}^{\hat{m}} \chi_{2 a, \rho} \varphi, \varphi\right)<c\left\|\chi_{2 a, \rho} \varphi\right\|^{2}
$$

for at least one $\varphi \in C_{0}^{2}\left(R_{0}\right), P^{\hat{m}} \varphi=\varphi$.

Let us prove that there is such a number $m_{0}$ that if $\left|m_{j_{0}}\right|>m_{0}$, for some $j_{0} \in$ $[1, \ldots, N+2], m_{j_{0}} \in \hat{m}$ inequality (A.2) does not hold.

For this goal we will use the next observation. Let $M=\max \left\{M_{1}, M_{2}\right\}$. For any $j \in[1, N+2]$ and $\varphi \in C_{0}^{2}\left(R_{0}\right)$ we have

$$
\left(T_{0}^{\hat{m}} \varphi, \varphi\right) \geqq M^{-1}\left\{\left\|\varphi_{\rho_{j}}^{\prime}\right\|^{2}+\left(\left(\frac{m_{j}}{\rho_{j}}+q_{j}|B| \rho_{j}\right)^{2} \varphi, \varphi\right)\right\} .
$$

It is clear that the kinetic energy is small only if all the particles are localized not far from the corresponding Landau orbits. These orbits have radiuses

$$
\rho_{j}=\left|m_{j}\right|^{1 / 2}|B|^{-1 / 2}\left|q_{j}\right|^{-1 / 2} \text {. }
$$

But if the total charge $Q$ is nonzero, $m$ is fixed and $m_{0} \rightarrow \infty$ the difference between the largest and the smallest Landau radiuses tends to $+\infty$. So the kinetic energy can not be small for all particles if the distances among particles are bounded.

Let $b>0$ be a positive number, $q_{j}$-be the charge of the particle with the number $j, q_{j}=Z_{j}, j=1,2, q_{j}=-1$ for $j>2 ; v(t), u(t) \in C^{2}\left(R_{+}^{1}\right), u^{2}+v^{2}=1, u=1$ if $t \in[0, b], u=0$ if $t>2 b ; u_{j}=u\left(\left.\left|\rho_{j}-\right| m_{j}\right|^{1 / 2}\left|q_{j}\right|^{-1 / 2}|B|^{-1 / 2} \mid\right), v_{j}=\left(1-u_{j}^{2}\right)^{1 / 2}$. It is easy to see that for any $\psi \in C_{0}^{2}\left(R_{0}\right)$,

$$
\left(T_{0}^{\hat{m}} \psi, \psi\right) \geqq\left(T_{0}^{\hat{m}} \psi u_{j}, \psi u_{j}\right)+\left(T_{0}^{\hat{m}} \psi v_{j}, \psi v_{j}\right)-c_{1}\left\|\psi u_{j}\right\|^{2}-c_{1}\left\|\psi v_{j}\right\|^{2},
$$

where $c_{1}=\max _{t}\left\{u_{t}^{\prime 2}(t)+v_{t}^{\prime 2}(t)\right\}$. If the number $b>0$ in the definition of the function $u$ is large, the second term of the right-hand part of (A.3) is large for $\varphi=\psi v_{j}$. Thus for large $b>0$ we have

$$
\left(T_{0}^{\hat{m}} \psi v_{j}, \psi v_{j}\right)-c_{1}\left\|\psi v_{j}\right\|^{2} \geqq c\left\|\psi v_{j}\right\|^{2} .
$$


Let $U=\prod_{j=1}^{N+2} u_{j}$ and $V=\left(1-U^{2}\right)^{1 / 2}$. It follows from (A.4) and (A.5) that

$$
\left(T_{0}^{\hat{m}} \psi, \psi\right) \geqq\left(T_{0}^{\hat{m}} \psi U, \psi U\right)-c_{1}\|\psi U\|^{2}+c\|\psi V\|^{2} .
$$

Denote by $\psi$ the function $\chi_{2 a, \rho} \varphi$, where $\varphi$ is a function for which (A.2) holds. At the support of the function $\chi_{2 a, \rho} \varphi \cdot U$ we have

$$
\left|\rho_{i}-\rho_{j_{0}}\right| \leqq 4 a \quad i=1, \ldots, N+2
$$

and

$$
\left.\left|\rho_{i}-\right| m_{i}\right|^{1 / 2}\left|q_{i}\right|^{-1 / 2}|B|^{-1 / 2} \mid \leqq 2 b .
$$

It follows from (A.7) and (A.8) that either $P^{\hat{m}} \psi U \equiv 0$ or for some $\alpha>0$, which does not depend on $\psi$, all $i=1,2, \ldots, N+2$ and large $m_{j_{0}}$.

$$
\left|m_{i}\right| \geqq \alpha\left|m_{j_{0}}\right| \geqq \alpha m_{0} \text {. }
$$

Hence if $m_{0}$ is large and $m_{i_{0}} q_{i_{0}}>0$ for at least one number $i_{0} \in[1, N+2]$,

$$
\left(T_{0}^{\hat{m}} \psi U, \psi U\right) \geqq\left(c+c_{1}\right)\|\psi U\|^{2},
$$

which completes the proof for this case.

Let us assume that $m_{i} q_{i}<0$ for $\forall i \in[1, N+2]$. At the support of the function $\chi_{2 a, \rho} U$ according to (A.7) and (A.8) we have

$$
\left.|| m_{i}\right|^{1 / 2}\left|q_{i}\right|^{-1 / 2}|B|^{-1 / 2}-\left|m_{j_{0}}\right|^{1 / 2}\left|q_{j_{0}}\right|^{-1 / 2}|B|^{-1 / 2} \mid \leqq 4(b+a),
$$

$i=1,2, \ldots, N+2$. So

$$
\left|m_{i} q_{i}^{-1}-m_{j_{0}} q_{j_{0}}^{-1}\right| \leqq 8(a+b)|B|^{1 / 2} \cdot\left|m_{j_{0}}\right|^{1 / 2}\left|q_{j_{0}}\right|^{-1 / 2}+16(a+b)^{2}|B| .
$$

It follows from (A.12) that

$$
\begin{aligned}
& m_{j_{0}} q_{j_{0}}^{-1} q_{i}-8(a+b)|B|^{1 / 2}\left|m_{j_{0}}\right|^{1 / 2}\left|q_{j_{0}}\right|^{-1 / 2} q_{i}-16(a+b)^{2}|B| q_{i} \\
& \leqq \\
& m_{i} \leqq m_{j_{0}} q_{j_{0}}^{-1} q_{i}+8(a+b)|B|^{1 / 2}\left|m_{j_{0}}\right|^{1 / 2}\left|q_{j_{0}}\right|^{-1 / 2} q_{i} \\
& \quad+16(a+b)^{2}|B| q_{i}
\end{aligned}
$$

for $i=1,2$ and

$$
\begin{aligned}
& m_{j_{0}} q_{j_{0}}^{-1} q_{i}+8(a+b)|B|^{1 / 2}\left|m_{j_{0}}\right|^{1 / 2}\left|q_{j_{0}}\right|^{-1 / 2} q_{i}+16(a+b)^{2}|B| q_{i} \\
& \leqq m_{i} \leqq m_{j_{0}} q_{j_{0}}^{-1} q_{i}-8(a+b)|B|^{1 / 2}\left|m_{j_{0}}\right|^{1 / 2}\left|q_{j_{0}}\right|^{-1 / 2} q_{i} \\
& \quad-16(a+b)^{2}|B| q_{i}
\end{aligned}
$$

for $i>2$, because $q_{i}>0$ for $i=1,2$ and $q_{i}<0$ for $i>2$. Taking a sum of inequalities (A.13) and (A.14) over $i=1, \ldots, N+2$ we get

$$
m_{j_{0}} q_{j_{0}}^{-1} Q-c_{2}\left|m_{j_{0}}\right|^{1 / 2}-c_{3} \leqq m \leqq m_{j_{0}} q_{j_{0}}^{-1} Q+c_{2}\left|m_{j_{0}}\right|^{1 / 2}+c_{3},
$$

where $Q=Z_{1}+Z_{2}-N$ is the total charge of the system and constants $c_{2}$ and $c_{3}$ do not depend on $\hat{m}$. It is clear that if $m_{j_{0}}$ large enough, $Q \neq 0$ and $m$ is fixed the inequality (A.15) does not hold. So in this case $\|\psi U\|=0$ and the statement of the lemma follows from (A.6).

Lemma is proved. 


\section{Appendix 2}

Let $C$ be a system of $p$ identical particles, $r_{i} i=1,2, \ldots, p$ be position vectors of these particles in the center of mass system of coordinates, $\sum_{i=1}^{p} r_{i}=0 ; \Delta_{0}$ be the Laplacian on the subspace

$$
\begin{gathered}
R_{0}=\left\{r_{0} \mid r_{0}=\left(r_{1}, \ldots, r_{p}\right), \sum_{i=1}^{p} r_{i}=0\right\} ; \\
\psi \in C_{0}^{2}\left(R_{0}\right) ; \quad \operatorname{supp}\{\psi\} \subset \Omega=\left\{r_{0}\left|r_{0} \in R_{0},\right| r_{i} \mid \leqq \frac{1}{2} L i=1, \ldots, p\right\} .
\end{gathered}
$$

Denote by $S$ the group of permutations of the particles.

Lemma A2.1. Let us assume that the function $\psi$ transforms according to an irreducible representation of the group $S$ corresponding to a Young diagram with one or two columns. Then one can find numbers $p_{0}>0$ and $\gamma>0$ (independent of $\psi$ ) such that for $p>p_{0}$,

$$
-\left(\Delta_{0} \psi, \psi\right) \geqq \gamma p^{5 / 3} L^{-2}\|\psi\|^{2}
$$

Proof. Let $r \in R^{3}, f(t) \in C_{0}^{2}[0,1], \int_{0}^{1} t^{2} f^{2}(t) d t=(4 \pi)^{-1},\left|f^{\prime}(t)\right| \leqq c_{1}, \bar{\psi}\left(r_{1} r_{0}\right)$ $=\psi\left(r_{0}\right) f\left(|r| \cdot L^{-1}\right)$. By $\tilde{\psi}\left(\tilde{r}_{1}, \ldots, \tilde{r}_{p}\right)$ we denote the function $\bar{\psi}$ as a function of $\tilde{r}_{i}=r_{i}+r, i=1, \ldots, p$. Function $\tilde{\psi}$ has the same permutational symmetry as $\psi$ and for it we have (see, for example, [4])

$$
-(\Delta \tilde{\psi}, \tilde{\psi}) \geqq \gamma_{1} p^{5 / 3} L^{-3}\|\psi\|^{2},
$$

where $\Delta$ is the Laplacian in $R^{3 p}, \gamma_{1}>0$.

Due to the equality

$$
-\Delta=-\Delta_{0}-p^{-1} \Delta_{r},
$$

we get

$$
\begin{aligned}
-(\Delta \tilde{\psi}, \tilde{\psi}) \cdot\|\tilde{\psi}\|^{-2}= & -\left(\Delta_{0} \psi, \psi\right) \cdot\|\psi\|^{-2} \\
& +p^{-1}\left\|\nabla_{r} f\left(|r| L^{-1}\right)\right\|^{2} \cdot\left\|f\left(|r| L^{-1}\right)\right\|^{-2}
\end{aligned}
$$

By the properties of function $f$

$$
\left\|f\left(|r| \cdot L^{-1}\right)\right\|^{2}=L^{3}
$$

and

$$
\left\|\nabla_{r} f\left(|r| L^{-1}\right)\right\|^{2} \leqq c_{1}^{2} L
$$

Hence

$$
-\left(\Delta_{0} \psi, \psi\right)\|\psi\|^{-2} \geqq\left(\gamma_{1} p^{5 / 3}-p^{-1} c_{1}^{2}\right) L^{-2} .
$$

The statement of lemma follows from (A.18). 


\section{Appendix 3}

In this section we obtain some estimates for the lowest eigenvalue of the kinetic energy operator for a system of $p$ particles localized in a compact region in a homogeneous magnetic field for $p \rightarrow \infty$.

The kinetic energy operator for this system has the form:

$$
T(p)=\sum_{j=1}^{p}\left\{\left(\frac{\partial}{\partial r_{j}^{(1)}}-i B r_{j}^{(2)}\right)^{2}+\left(\frac{\partial}{\partial r_{j}^{(2)}}+i B r_{j}^{(1)}\right)^{2}-\frac{\partial^{2}}{\partial r_{j}^{(3)^{2}}}\right\}
$$

where $r_{j}=\left(r_{j}^{(1)}, r_{j}^{(2)}, r_{j}^{(3)}\right)$ are the coordinates of electrons. Let each electron be localized in a ball with the radius $d / 2$ :

$$
\left|r_{j}\right| \leqq \frac{d}{2} \quad j=1,2, \ldots, p ; d>0
$$

We shall study the operator $T(p)$ in the space $\mathscr{L}_{2}\left(R^{3 p}\right)$ with the Dirichlet boundary conditions at $\left|r_{j}\right|=d / 2$. Let us assume that the magnetic field is strong: $d>2 B^{-1 / 2}$.

Denote by $S$ the group of permutations of electrons, $\alpha$ be a type of irreducible representation of the group $S$, satisfying the Pauli principle. Let $\sigma=(\alpha, m, \omega)$, where $m$ is a weight of the representation of the $S O(2)$ group and $\omega$ is a parity with respect to reflections of the third axis. We denote by $P^{\sigma}$ the projector in $\mathscr{L}_{2}\left(R^{3 p}\right)$ onto the subspace of functions with symmetry $\sigma$.

Theorem A3. One can find a number $\gamma_{2}>0$ such that the lowest nonzero eigenvalue $\lambda^{\sigma}$ of the operator $T P^{\sigma}$ in the region $\left|r_{i}\right| \leqq d / 2$ with the Dirichlet boundary conditions for all $p>0$ satisfies the inequality

$$
\lambda^{\sigma} \geqq \min \left\{2 B p, B p+\gamma_{2} p^{3} B^{-3} d^{-8}\right\} \text {. }
$$

Let

$$
\begin{gathered}
\Omega=\left\{\left.r\left|\left(r^{(1)}, r^{(2)}\right) \in R^{2},\right| r^{(1)}\right|^{2}+\left|r^{(2)}\right|^{2} \leqq \frac{d^{2}}{4}\right\}, \\
T_{\perp}^{1}=\left(i \frac{\partial}{\partial r^{(1)}}-B r^{(2)}\right)^{2}+\left(i \frac{\partial}{\partial r^{(2)}}+B r^{(1)}\right)^{2}
\end{gathered}
$$

be the operator in $\Omega$ with the Dirichlet boundary conditions at

$$
\left\{\left|r^{(1)}\right|^{2}+\left|r^{(2)}\right|^{2}\right\}^{1 / 2}=\frac{d}{2}
$$

$N(\lambda)$ be the number of eigenvalues of the operator $T_{\perp}^{1}$ (with regard to their multiplicity) which are less or equal to $\lambda$. The proof of Theorem A3 relies upon.

Lemma A3.1. The next inequality holds

$$
N(4 B) \leqq 52 B d^{2} .
$$


Proof of Lemma A3.1. Let us make a partition of the region $\Omega$ into subregions $\Omega_{s}$ by the lines

$$
r^{(i)}=k \cdot B^{-1 / 2} \quad k=0, \pm 1, \pm 2, \ldots \quad i=1,2,
$$

the number of such regions is not greater than $4 d^{2} B$.

Let $\Omega_{S_{0}}$ be a fixed subregion. It follows from variational reasons that to prove the lemma it suffices to construct 13 functions $\varphi_{1}, \ldots, \varphi_{13}$ such that

1) $\varphi_{l}(r)=0$ for $r \notin \Omega_{S_{0}} l=1, \ldots, 13$;

2) for any $\psi \in C_{0}^{2}(\Omega), \psi \perp \varphi_{l} l=1, \ldots, 13$,

$$
\begin{aligned}
J[\psi] & \equiv \int_{\Omega_{S_{0}}}\left\{\left|i \frac{\partial \psi}{\partial r^{(1)}}-B r^{(2)} \psi\right|^{2}+\left|i \frac{\partial \psi}{\partial r^{(2)}}+B r^{(1)} \psi\right|^{2}\right\} d \Omega \\
& \geqq 4 B \int_{\Omega_{S_{0}}}|\psi|^{2} d \Omega .
\end{aligned}
$$

Let the region $\Omega_{S_{0}}$ be given by inequalities

$$
\left|r^{(1)}-r_{0}^{(1)}\right| \leqq \frac{1}{2} B^{-1 / 2}, \quad\left|r^{(2)}-r_{0}^{(2)}\right| \leqq \frac{1}{2} B^{-1 / 2},
$$

$\left(r_{0}^{(1)}, r_{0}^{(2)}\right)$ be the center of the region and $\psi_{1}=\psi \cdot \exp \left\{i B\left(r_{0}^{(1)} r^{(2)}-r_{0}^{(2)} r^{(1)}\right)\right\}$. It is easy to see that

$$
J[\psi]=J_{1}\left[\psi_{1}\right],
$$

where

$$
\begin{aligned}
J_{1}\left[\psi_{1}\right] & =\int_{\Omega_{S_{0}}}\left\{\left|i \frac{\partial \psi_{1}}{\partial r^{(1)}}-B\left(r^{(2)}-r_{0}^{(2)}\right) \psi_{1}\right|^{2}+\left|i \frac{\partial \psi_{1}}{\partial r^{(2)}}+B\left(r^{(1)}-r_{0}^{(1)}\right) \psi_{1}\right|^{2}\right\} d \Omega \\
& \geqq \frac{1}{2} \int_{\Omega_{S_{0}}}\left\{\left|\frac{\partial \psi_{1}}{\partial r^{(1)}}\right|^{2}+\left|\frac{\partial \psi_{1}}{\partial r^{(2)}}\right|^{2}\right\} d \Omega-B^{2} \cdot B^{-1} \int_{\Omega_{S_{0}}}|\psi|^{2} d \Omega .
\end{aligned}
$$

Let $\varphi_{1}^{0}, \ldots, \varphi_{13}^{0}$ be the first 13 eigenfunctions for the Laplacian in the region $\Omega_{S_{0}}$ with the Neumann boundary conditions. If $\psi_{1} \perp \varphi_{l}^{0} l=1, \ldots, 13$, we have

$$
\int_{\Omega_{S_{0}}}\left\{\left|\frac{\partial \psi_{1}}{\partial r^{(1)}}\right|^{2}+\left|\frac{\partial \psi_{1}}{\partial r^{(2)}}\right|^{2}\right\} \geqq 10 B \int_{\Omega_{S_{0}}}\left|\psi_{1}\right|^{2} d \Omega,
$$

and according to (A.24),

$$
J_{1}\left[\psi_{1}\right] \geqq 4 B \int_{\Omega_{S_{0}}}\left|\psi_{1}\right|^{2} d \Omega
$$

Hence the inequality (A.23) holds for

$$
\varphi_{l}=\varphi_{l}^{0} \cdot \exp \left\{-i B\left(r_{0}^{(1)} r^{(2)}-r_{0}^{(2)} r^{(1)}\right)\right\} .
$$

The lemma is proved.

Proof of Theorem A3. Let $r_{i}^{\perp}=\left(r_{i}^{1}, r_{i}^{2}\right)$ be the projection of a vector $r_{i}$ onto the plane orthogonal to the direction of the magnetic field, $T^{0}$ be the operator defined 
by (A.19) in the region

$$
\left|r_{i}^{(3)}\right| \leqq \frac{d}{2}, \quad\left|r_{i}^{\perp}\right| \leqq \frac{d}{2}
$$

with the Dirichlet boundary conditions.

Notice that the region (A.27) contains the ball (A.20). Thus the lowest eigenvalue $\lambda_{0}^{\sigma}$ of the operator $T^{0} P^{\sigma}$ may be only less than the same one for $T P^{\sigma}$. Let us estimate the lower bound for $\lambda_{0}^{\sigma}$.

For an arbitrary eigenspace of $T^{0}$ functions $\psi$,

$$
\psi=\prod_{i=1}^{p} \varphi_{s(i)}\left(r_{i}^{(1)}, r_{i}^{(2)}\right) f_{t(i)}\left(r_{i}^{(3)}\right),
$$

where $\varphi_{s(i)}$ are the orthonormalized eigenfunctions of the operator $T_{\perp}^{1}$ and $f_{t(i)}$ are the orthonormalized eigenfunctions of the operator

$$
\frac{\partial^{2}}{\partial\left(r^{(3)}\right)^{2}}
$$

in the region $\left|r^{(3)}\right| \leqq d / 2$ with the Dirichlet conditions may be chosen as a basis.

Let us prove that if for some $\lambda$, a corresponding eigenfunction $\psi_{0}$ of the operator $T^{0}$ satisfies the inequality $P^{\sigma} \psi_{0} \neq 0$, then

$$
\lambda \geqq \min \left\{2 B p, B p+\gamma_{2} p^{3} B^{-3} d^{-8}\right\}
$$

Notice that function $\psi_{0}$ is a linear combination of functions (A.28). Consequently among these functions there is a function $\psi$ such that $T^{0} \psi=\lambda \psi$ and $P^{\sigma} \psi \neq 0$.

Assume that among the function $\varphi_{s(i)}$ at the representation (A.28) for $\psi$ there are at least $p / 2$ functions corresponding to the eigenvalues of the operator $T_{\perp}^{1}$ which are greater than $4 B$. Then $\lambda \geqq 2 B p$.

Contrarily, let the function $\psi$ has at least $p / 2$ functions $\varphi_{s(i)}$, corresponding to the eigenvalues of $T_{\perp}^{1}$ which are less than $4 B$, in its representation (A.28). Due to Lemma A3.1 operator $T_{\perp}^{1}$ has less than $52 B d^{2}$ such orthonormalized eigenfunctions. Hence to satisfy the Pauli principle (the condition $P^{\sigma} \psi \neq 0$ ) the function $\psi$ must have at least $4^{-1} p\left(52 B d^{2}\right)^{-1}$ different functions $f_{t(i)}$ in (A.28). It is easy to see that for small $\gamma_{2}>0$, the sum of $208^{-1} p B^{-1} d^{-2}$ lowest eigenvalues of the operator

$$
-\frac{\partial^{2}}{\partial\left(r^{(3)}\right)^{2}}
$$

with the Dirichlet boundary conditions at $\left|r^{(3)}\right|=d / 2$ is greater than $\gamma_{2} p^{3} B^{-3} d^{-8}$.

Notice that inf $T_{\perp}^{1}=B$ and consequently $\lambda \geqq B p+\gamma_{2} p^{3} B^{-3} d^{-8}$, which completes the proof of the theorem. ing his visit to Trondheim, Professor T. Hoffmann-Ostenhoff for the invitation to the International Erwin Schrödinger Institute and Professor B Ruskai for a large number of useful remarks. 


\section{References}

1 Avron, J E, Herbst, I W, Simon, B : Schrödinger Operators with Magnetic Fields III Atoms in Magnetic Fields Commun. Math. Phys 79, 529-572 (1981)

2 Cycon, H L, Froese, R G, Kirsh, W, Simon, B.: Schrödinger Operators Berlin-HeidelbergNew York: Springer-Verlag, 1987

3 Jörgens, K, Weidmann, J : Spectral Properties of Hamiltonian Operators Berlin-Heidelberg-New York: Springer-Verlag, 1973

4 Lieb, E., Simon, B: The Thomas Fermi Theory of Atoms Molecules and Solids Adv Math 23, 22-116 (1977)

5. Ruskai, M B : Limits on Stability of Positive Molecular Ions Lett Math Physics 18, 121-132 (1989)

6 Vugalter, S., Zhislin, G: On the Localization of the Essential Spectrum of $n$-Particle Hamiltonians in Magnetic Field Theoret and Math Physics 97, 94-112 (1993)

7 Zhislin, G : Spectrum of Differential Operators of Quantum Mechanical Many-Particle Systems in Spaces of Functions of a Given Symmetry Izv AN SSSR (Ser Math ) 33, 590-649 (1969)

Communicated by B Simon 
